\title{
Locality of Conformal Fields in Two Dimensions: Exchange Algebra on the Light-Cone
}

\author{
Karl-Henning Rehren \\ FU Berlin, Institut für Theorie der Elementarteilchen, Arnimallee 14, D-1000 Berlin 33
}

\begin{abstract}
We discuss the exchange algebra of light-cone operators as the fundamental structure of two-dimensional conformal quantum field theory. It is necessary in order to account for the locality properties of Wightman functions of conformal fields. We discuss the consistency requirements of this new type of algebra, and obtain a classification containing the well known "minimal models".
\end{abstract}

\section{Introduction}

Belavin, Polyakov, Zamolodchikov [1] have classified conformal quantum field theories in two dimensions, which contain only a finite number of "primary" fields. They introduced an algorithm, based on Ward identities associated with the Euclidean conformal invariance, in order to calculate correlations as solutions to certain differential equations.

Let us concentrate on a peculiar feature of these $n$-point functions. They have the factorized structure of a bilinear form in so-called conformal block functions depending on the coordinates of one light-cone only. It appears as a little miracle that these blocks can be combined in such a manner, that the full $n$-point function satisfies the requirements of locality. The reason lies in remarkable functional properties of the conformal block functions.

The aim of the present study is to identify the relevant operator algebra which lies at the origin of these properties [2]. The operators involved in this "exchange algebra" are intertwining light-cone operators interpolating between different representation sectors of the Virasoro algebra. The study of the intertwining operators should be most important for the understanding of the highly nontrivial interrelations among the Rocha-Caridi characters [3] of different Virasoro Verma modules. These interrelations are essential for the existence of modular invariant partition functions [4]. The existence of interpolating fields is no completely new issue. Actually they have been discussed in the old days of conformal invariance in 
order to resolve the causality problem associated with the transition from Euclidean to Minkowski conformal quantum field theory [5].

In Sect. 2 we discuss the analytic continuation properties of conformal block functions necessary to satisfy the requirement of locality in the full $n$-point function. In Sect. 3 we review the origin of light-cone factorization of local $n$-point functions in terms of global vacuum expansions of operator products of conformal Minkowski fields. We introduce intertwining light-cone operators and the exchange algebra they fulfill, thus explaining at a more fundamental level the analytic properties of conformal blocks. In Sect. 4 we discuss the associativity of exchange algebras and obtain a (partial) classification of such algebras, which are related with known models in Sect. 5.

On more general grounds (e.g. considering non-observable fields like soliton operators, which are nonlocal relative to each other), a bilinear operator algebra relating different operator orderings by means of some "statistics matrix" has been discussed independently by Fröhlich [6]. His analysis of associativity leads to the same type of conditions (intimately related with the Yang-Baxter equation and "braid groups") on the statistics matrices, as ours on the exchange matrices. Fröhlich's concept applies to non-conformal quantum field theory (statistical mechanics) as well, where clearly the peculiar features of light-cone factorization are absent. It is only due to the latter that the (anti-)commutation of local observables can be discussed in terms of more fundamental non-trivial algebras of relatively nonlocal "constituent" fields (i.e. exchange algebras of light-cone fields).

\section{Analytic Continuation of Conformal Blocks}

In the Euclidean formalism of ref. [1] it has been derived from conformal Ward identities that a correlation function of primary fields solves a set of real linear differential equations. These are equations in the light-cone variables of either lightcone separately. Hence the most general structure of a local $n$-point function is

$$
\left\langle\Phi_{1}\left(x_{1}\right) \ldots \Phi_{n}\left(x_{n}\right)\right\rangle=\sum_{\alpha=1}^{r} F_{\alpha}(u) \bar{F}_{\alpha}(v) \equiv(\bar{F} \cdot F),
$$

where $r$ denotes the number of independent solutions to these differential equations. We shall in the next section sketch the argument explaining the structure of Eq. (1) in a formalism applying directly to local fields in Minkowski space.

In Eq. (1), $u(v)$ denote all light-cone variables of the $u=x+t(v=x-t)$ lightcone and $F_{\alpha}(u)\left(\bar{F}_{\alpha}(v)\right)$ are the solutions to the $u(v)$ differential equations. Except for some appropriate common factors $\Pi u_{i j}^{\delta_{i j}}\left(\Pi v_{i j}^{\bar{\delta}_{j}}\right)$, where $u_{i j}=u_{i}-u_{j}\left(v_{i j}=v_{i}-v_{j}\right)$, these functions depend only on anharmonic ratios of light-cone variables. Except for a common overall complex phase factor depending on the statistics of the fields involved, they are real in the ordered regions $u_{i}>u_{i+1}, v_{i}>v_{i+1}$ of their respective arguments. With a slight abuse of the notion introduced in [1] we shall refer to $F_{\alpha}(u)$ and $\bar{F}_{\alpha}(v)$ as conformal block functions.

How can this structure be compatible with locality of quantum fields in Minkowski space? Consider the case $\Phi_{i}=\Phi_{i+1}=\Phi$. Compare $\left\langle\ldots \Phi\left(x_{i}\right) \Phi\left(x_{i+1}\right) \ldots\right\rangle$ with $\left\langle\ldots \Phi\left(x_{i+1}\right) \Phi\left(x_{i}\right) \ldots\right\rangle$. The latter can be obtained from 
the former by analytic continuation from the configuration point $\left(\ldots, x_{i}, x_{i+1}, \ldots\right)$ to the permuted point $\left(\ldots, x_{i+1}, x_{i}, \ldots\right)$ with appropriate $i \varepsilon$-prescriptions for the variables $u_{i i+1}$ and $v_{i i+1}$ of the functions $F_{\alpha}$ and $\bar{F}_{\alpha}$. On the other hand, locality requires that the full $n$-point function does not change under the exchange $x_{i+1} \leftrightarrow x_{i}$ if $u_{i i+1} v_{i i+1}=-\left(x_{i}-x_{i+1}\right)^{2}>0$. This requirement is compatible with the light-cone factorized structure of the $n$-point function only if under analytic continuation

and

$$
\begin{aligned}
& F_{\alpha}\left(\ldots u_{i+1}, u_{i} \ldots\right)=\eta \sum_{\beta} A_{\alpha \beta} F_{\beta}\left(\ldots u_{i}, u_{i+1} \ldots\right) \text { for } u_{i i+1}>0, \\
& \bar{F}_{\alpha}\left(\ldots v_{i+1}, v_{i} \ldots\right)=\bar{\eta} \sum_{\beta} B_{\alpha \beta} \bar{F}_{\beta}\left(\ldots v_{i}, v_{i+1} \ldots\right) \text { for } v_{i i+1}>0
\end{aligned}
$$

The phase factors $\eta=e^{2 \pi i d}$ and $\bar{\eta}=e^{-2 \pi i \bar{d}}$, where $d, \bar{d}$ are the light-cone dimensions of the field $\Phi$, have been split off for convenience. For bosonic fields they cancel each other under simultaneous analytic continuation on both light-cones, i.e. analytic continuation in the Minkowski variables. For fermionic fields they account for the anticommutation minus sign.

As long as Eq. (3) holds, the matrices $A$ and $B$ relevant for the exchange of $x_{i+1} \leftrightarrow x_{i}$ cannot depend on the coordinates: $A$, describing the analytic continuation of some functions of the $u$ 's, can depend only on the $u$ 's, and $B$ can depend only on the $v$ 's; then $B=\left(A^{-1}\right)^{T}$ implies that they are both constant matrices.

Equation (3) ceases to hold if either $u_{i i+1}$ or $v_{i i+1}$ changes sign; then the separation of the two Minkowski points is no longer space-like. It holds again if both $u_{i i+1}$ and $v_{i i+1}$ are negative. Thus $A$ and $B$ may depend on the signs of $u_{i i+1}$ and $v_{i i+1}$ respectively.

By definition, $F_{\alpha}\left(\ldots u_{i+1}, u_{i}, \ldots\right)$ is the analytic continuation of $F_{\alpha}\left(\ldots, u_{i}, u_{i+1}, \ldots\right)$ such that the new value $u_{i}$ of the right-hand argument $\left(u_{i+1}\right.$ of the left-hand argument) is approached from the upper (lower) complex half-plane. Thus, for $u_{i i+1}>0, u_{i}$ and $u_{i+1}$ surround each other with clockwise orientation. For $u_{i i+1}<0$, the orientation is reversed, hence the analytic continuation is described by the inverse matrices

$$
\begin{aligned}
& F_{\alpha}\left(\ldots u_{i+1}, u_{i} \ldots\right)=\eta^{-1} \sum_{\beta}\left(A^{-1}\right)_{\alpha \beta} F_{\beta}\left(\ldots u_{i}, u_{i+1} \ldots\right) \text { for } u_{i i+1}<0, \\
& \bar{F}_{\alpha}\left(\ldots v_{i+1}, v_{i} \ldots\right)=\bar{\eta}^{-1} \sum_{\beta}\left(B^{-1}\right)_{\alpha \beta} \bar{F}_{\beta}\left(\ldots v_{i}, v_{i+1} \ldots\right) \text { for } v_{i i+1}<0 .
\end{aligned}
$$

Since $F_{\alpha}$ and $\bar{F}_{\alpha}$ are real functions (except for a common phase) in the ordered region of their arguments, we must have

$$
A^{-1}=A^{*} \text { and } B^{-1}=B^{*} .
$$

If $\Phi$ is a hermitian, scalar field, $F_{\alpha}$ and $\bar{F}_{\alpha}$ coincide in the ordered region of their arguments, but are taken on different Riemann sheets outside, since the $i \varepsilon$ prescription on the $v$ light-cone is opposite to that on the $u$ light-cone. In that case we have in addition:

$$
B=A^{*},
$$

which implies together with Eqs. (3), (4) that $A$ is a symmetric unitary matrix. 
The effect of the various light-cone exchange operations on an $n$-point function can be visualized by the following diagram:

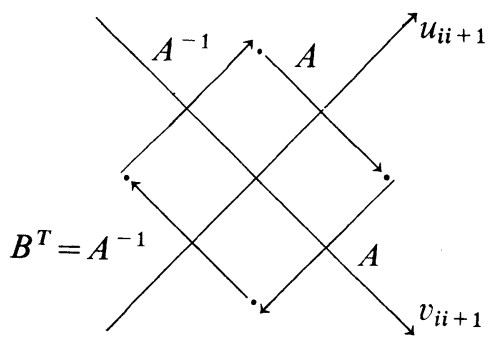

The analytic continuation from a space-like separation $x$ to $-x$ takes $(\bar{F} \cdot F)$ into $\left(\bar{F} A^{-1} \cdot A F\right)=(\bar{F} \cdot F)$, while the analytic continuation from a forward or backward time-like separation $x$ to $-x$ takes $(\bar{F} \cdot F)$ into $(\bar{F} A \cdot A F)$ or $\left(\bar{F} A^{-1} \cdot A^{-1} F\right)$ respectively. In particular, a true interaction requires nontrivial exchange matrices $A$.

We have arrived at the remarkable conclusion that the analytic exchange of two neighbouring light-cone coordinates within a conformal block of an $n$-point function amounts to a mixing of all conformal blocks contributing to the local $n$ point function in question with a matrix $A=A^{*-1}$ depending on the two light-cone coordinates to be exchanged only by their relative sign, and depending on the other light-cone coordinates of the $n$-point function not at all. This suggests that the relevant algebra implying Eqs. (2) and (2') is an "exchange algebra" involving just a pair of "light-cone operators", which reside at the two light-cone points to be exchanged. The next section will explain what this type of algebra looks like precisely.

\section{Intertwining Operators and Exchange Algebra}

Finite special conformal transformations of Minkowski space can take space-like separations into time-like separations. Hence, fields that transform irreducibly under all conformal transformations cannot commute with each other at space-like distances without commuting at time-like distances. This is the Einstein causality paradox. It has been solved by the observation that in the quantum field theory only the covering of the classical conformal group is represented, and that local fields transform irreducibly only under Poincaré and scale transformations, but not under special conformal transformations [5].

The conformal group contains a compact subgroup $\mathbf{U}(1) \otimes \mathbf{U}(1)$ generated by the "conformal Hamiltonians" $L_{0}$ and $\bar{L}_{0}$. Its elements act like rotations on the unit circle, if the light-cone (= real axis) is mapped by a Möbius transformation onto the unit circle. The symmetry of the quantum theory is the covering of the conformal group, i.e. the rotations by $2 \pi$ are represented by nontrivial operators $Z=e^{-2 \pi i L_{0}}, \bar{Z}$ $=e^{2 \pi i \bar{L}_{0}}$. In the "heaven and hell" picture [7] the unit circle is unwrapped into a $2 \pi$ interval of the real axis, such that a local field resides on a covering of the Minkowski world. $Z$ and $\bar{Z}$ transform the local field irreducibly, shifting however its (compactified) light-cone coordinate by $2 \pi$ into another sheet. 
We prefer to avoid the "heavens and hells", working with the following equivalent picture [5]. The copies of the local field living in other sheets are traded for a Fourier decomposition of the field with respect to the central elements $Z$ and $\bar{Z}$. Let us describe this in modern language. $Z, \bar{Z}$ are diagonalized with eigenvalues $e^{-2 \pi i h}, e^{2 \pi i \bar{h}}$ on sectors $\mathscr{H}_{\Phi}$ of the Hilbert space which are generated from the vacuum by primary fields $\Phi$ and their descendents with light-cone dimensions $d=h \bmod \mathbb{Z}, \bar{d}=\bar{h} \bmod \mathbb{Z}$. The sector $\mathscr{H}_{\Phi}$ is a representation of two independent Virasoro algebras generated by the two light-cone components $T(u)$ and $\bar{T}(v)$ of the energy-momentum tensor field, and has therefore the structure of a direct product of two Verma modules $\mathscr{H}_{\Phi} \equiv \mathscr{H}_{H}=V_{h} \otimes V_{\bar{h}}$, where $H=(h, \bar{h})$ are the light-cone dimensions of the primary field $\Phi=\Phi_{h \bar{h}} \equiv \Phi_{H}$. (The notion "Verma module" is strictly accurate 'only in the Euclidean formalism of [1]. We prefer to denote in the following $V_{h}$ and $V_{h}^{-}$by " $T$ - and $\bar{T}$-modules", instead.)

The spectral decomposition of a local conformal field with respect to $Z$ and $\bar{Z}$ yields a number of "nonlocal parts":

$$
\Phi_{d \bar{d}}(x)=\sum_{\xi \xi} \Phi_{d \bar{d}}^{\xi \bar{\xi}}(x)
$$

The values of the numbers $\xi, \bar{\xi} \in \mathbb{R} \bmod \mathbb{Z}$ contributing to this sum depend on the interactions of the field $\Phi$. In contrast with the local field, its nonlocal parts transform irreducibly under conformal transformations. The transformation law for special transformations exhibits a complex phase behavior determined by the $Z, \bar{Z}$ transformation laws,

$$
Z^{n} \bar{Z}^{\bar{n}} \Phi_{d \bar{d}}^{\xi \bar{\xi}}(x) \bar{Z}^{-\bar{n}} Z^{-n}=e^{-2 \pi i n(d-\xi)} e^{2 \pi i \bar{n}(\bar{d}-\bar{\xi})} \Phi_{d \bar{d}}^{\xi \bar{\xi}}(x)
$$

For more detailed formulae we refer the reader to ref. [8].

From Eq. (7) we deduce the selection rules

$$
\left\langle\Phi_{d_{1} \bar{d}_{1}} \Phi_{d \bar{d}}^{\xi \bar{\xi}} \Phi_{d_{3} \bar{d}_{3}}\right\rangle \neq 0 \quad \text { only if } \quad \xi=d-d_{1}+d_{3} \bmod \mathbb{Z}, \quad \bar{\xi}=\bar{d}-\bar{d}_{1}+\bar{d}_{3} \bmod \mathbb{Z} .
$$

We shall thus consider the nonlocal part $\Phi_{d d}^{\xi \bar{\xi}}$ as an "interpolating field", and denote it by $(\Phi)_{H_{1} H_{3}}: \mathscr{H}_{H_{3}} \rightarrow \mathscr{H}_{H_{1}} ;\left(h_{i}, \bar{h}_{i}\right)=\left(d_{i}, \bar{d}_{i}\right)$ mod $\mathbb{Z}$. Take as an example the Ising model. The Hilbert space is the orthogonal sum of three sectors $\mathscr{H}=\mathscr{H}_{1} \oplus \mathscr{H}_{\sigma} \oplus \mathscr{H}_{\varepsilon}$. The order field $\sigma$ has four nonlocal parts $\sigma_{\sigma 1}: \mathscr{H}_{1}$ $\rightarrow \mathscr{H}_{\sigma}, \sigma_{\varepsilon \sigma}: \mathscr{H}_{\sigma} \rightarrow \mathscr{H}_{\varepsilon}$, and $\sigma_{1 \sigma}=\sigma_{\sigma 1}^{+}, \sigma_{\sigma \varepsilon}=\sigma_{\varepsilon \sigma}^{+}$.

In an $n$-point function $\left\langle\Phi_{1} \ldots \Phi_{n}\right\rangle$ only the part $\left(\Phi_{1}\right)_{0 H_{1}}$ of $\Phi_{1}$ and the part $\left(\Phi_{n}\right)_{H_{n} 0}$ of $\Phi_{n}$ contribute. For $n>3$ every local field "in the middle" will in general have several nonvanishing contributions. Thus the local $n$-point function is a sum of terms where each term corresponds to one chain of interpolating fields leading from the vacuum sector $\mathscr{H}_{1}$ back to the vacuum sector; e.g. $\langle\sigma \sigma \sigma \sigma\rangle=\left\langle\sigma_{1 \sigma} \sigma_{\sigma 1} \sigma_{1 \sigma} \sigma_{\sigma 1}\right\rangle$ $+\left\langle\sigma_{1 \sigma} \sigma_{\sigma \varepsilon} \sigma_{\varepsilon \sigma} \sigma_{\sigma 1}\right\rangle$.

An operator product $\left(\Phi_{1}\right)_{H^{\prime} H_{2}}\left(x_{1}\right) \Phi_{2}\left(x_{2}\right)|0\rangle$ of two nonlocal parts acting on the vacuum state can be globally expanded into an integral over states $\Phi^{\prime}\left(x^{\prime}\right)|0\rangle$. The integral extends over the entire Minkowski space [5,8]. The integral kernels factorize into a product of two kernels, either one depending on the coordinates (and the dimensions $d$ and phases $\xi$ ) of one light-cone only. It is seen by repeated 
application of this vacuum expansion that then every chain contributing to the local $n$-point function factorizes, too:

$$
\left\langle\left(\Phi_{1}\right)_{0 H_{1}}\left(x_{1}\right)\left(\Phi_{2}\right)_{H_{1} H^{\prime}}\left(x_{2}\right) \ldots\left(\Phi_{n}\right)_{H_{n} 0}\left(x_{n}\right)\right\rangle=F_{\alpha}(u) \cdot \bar{F}_{\alpha}(v) .
$$

This light-cone factorization allows us to represent the interpolating fields (=nonlocal parts) as products of independent light-cone operators:

$$
(\Phi)_{H_{1} H_{2}}(x)=a_{h_{1} h_{2}}^{(\Phi)}(u) \cdot \bar{a}_{h_{1} h_{2}}^{(\Phi)}(v) ; \quad a_{h_{1} h_{2}}^{(\Phi)}: V_{h_{2}} \rightarrow V_{h_{1}}, \quad \bar{a}_{h_{1} \bar{h}_{2}}^{(\Phi)}: V_{h_{2}}^{-} \rightarrow V_{\bar{h}_{1}}^{-},
$$

where $V_{h}, V_{\bar{h}}$ are the $T, \bar{T}$-modules introduced above. Every conformal block $F_{\alpha}$ corresponds to one chain of light-cone operators:

$$
F_{\alpha}(u)=\left\langle a_{0 h_{1}}^{\left(\Phi_{1}\right)}\left(u_{1}\right) a_{h_{1} h^{\prime}}^{\left(\Phi_{2}\right)}\left(u_{2}\right) \ldots a_{h_{n} 0}^{\left(\Phi_{n}\right)}\left(u_{n}\right)\right\rangle .
$$

For the Ising field $\sigma$ we have to introduce four operators on either light-cone: $a(u): V_{0} \rightarrow V_{1 / 16}, b(u): V_{1 / 16} \rightarrow V_{1 / 2}, a^{+}(u), b^{+}(u) ; \quad$ and $\bar{a}(v), \bar{b}(v), \bar{a}^{+}(v), \bar{b}^{+}(v)$.

These operators are the intertwining operators mentioned in the introduction. Their algebra clearly is no commutator algebra of local fields. Instead they fulfill an exchange algebra to be specified in an instant, which implies the analytic continuation properties Eqs. (2), (2') of the functions $F_{\alpha}$ [Eq. (11)]. In turn, as we have seen in the preceding section, these properties guarantee the locality of full $n$ point functions of the local primary fields.

Consider now, in order to study the exchange algebra of a field $\Phi$, the conformal blocks $F_{\alpha}(u)$ contributing to a 4 -point function $\left\langle\Phi_{1} \Phi \Phi \Phi_{4}\right\rangle . F_{\alpha}(u)$ is uniquely associated with an "intermediate" $T$-module $V_{h_{\alpha}}$ :

$$
F_{\alpha}(u)=\left\langle a_{0 h_{1}}^{\left(\Phi_{1}\right)}\left(u_{1}\right) \cdot a_{h_{1} h_{\alpha}}^{(\Phi)}\left(u_{2}\right) a_{h_{\alpha} h_{4}}^{(\Phi)}\left(u_{3}\right) \cdot a_{h_{4} 0}^{\left(\Phi_{4}\right)}\left(u_{4}\right)\right\rangle .
$$

The algebra Eq. (2) describing the analytic exchange of the variables $u_{2}, u_{3}$ can now be translated into the exchange algebra satisfied by intertwining operators:

$$
a_{h_{1} h_{\alpha}}^{(\Phi)}\left(u_{3}\right) a_{h_{\alpha} h_{4}}^{(\Phi)}\left(u_{2}\right)=\eta \sum_{\beta} A_{\alpha \beta} a_{h_{1} h_{\beta}}^{(\Phi)}\left(u_{2}\right) a_{h_{\beta} h_{4}}^{(\Phi)}\left(u_{3}\right) \quad \text { for } \quad u_{2}>u_{3}
$$

Clearly, the matrix $A$ (even its size) depends on the choice of the two "external" $T$-modules $V_{h_{1}}$ and $V_{h_{4}}$.

As an example we give the exchange algebra of the Ising field $\sigma$ [2]:

$$
\begin{aligned}
a^{+}\left(u^{\prime}\right) a(u) & =e^{i \pi / 8} \quad a^{+}(u) a\left(u^{\prime}\right) \\
b\left(u^{\prime}\right) a(u) & =e^{i \pi / 8}(-i) b(u) a\left(u^{\prime}\right) \\
\left(\begin{array}{ll}
a\left(u^{\prime}\right) & a^{+}(u) \\
b^{+}\left(u^{\prime}\right) b(u)
\end{array}\right) & =e^{i \pi / 8} \frac{1}{\sqrt{2}}\left(\begin{array}{ll}
e^{-i \pi / 4} e^{i \pi / 4} \\
e^{i \pi / 4} & e^{-i \pi / 4}
\end{array}\right)\left(\begin{array}{ll}
a(u) & a^{+}\left(u^{\prime}\right) \\
b^{+}(u) b\left(u^{\prime}\right)
\end{array}\right) \\
b\left(u^{\prime}\right) b^{+}(u) & =e^{i \pi / 8} \quad b(u) b^{+}\left(u^{\prime}\right) \text { for } u>u^{\prime} .
\end{aligned}
$$

For intertwining operators belonging to different local fields, the argument is similar. The exchange algebra gives the product of two intertwining operators at $u^{\prime}, u$ belonging to the respective fields as a linear combination of products of intertwining operators at $u, u^{\prime}$ belonging to the same fields in the reversed order. A 
mixing is only possible among operator products with common initial and final $T$-modules.

The important point is that the exchange matrices describing the analytic continuation of 4-point conformal blocks must also determine the analytic continuation behaviour of all higher $n$-point conformal blocks. In practice, neither the Ward identity differential equations can be solved for $n>4$, nor seem the (very restrictive) analytic continuation properties alone to be helpful for the explicit determination of $n>4$ conformal blocks. However, combining the analytic continuation properties with the order/disorder duality and with the knowledge of equal-time correlations, all mixed order/disorder $2 n$-point functions of the Ising model have been determined explicitly [2].

In the next section we shall discuss intrinsically the associativity conditions on the exchange algebra coefficients, comparable with the Jacobi identity for a Lie algebra. In the present case the structure of these conditions turns out to be that of a braid algebra [9]. In solving the conditions in a typical situation, we shall see that even the 4-point conformal blocks need not to be known explicitly. Some of the exchange matrix coefficients can, with the help of 3-point functions alone, be computed from the dimensions of the fields involved, while the remaining ones are determined by consistency.

\section{Associativity of the Exchange Algebra}

We have argued in Sect. 2 that the transposition $(i, i+1)$ of two neighbouring coordinates $u_{i}, u_{i+1}$ within a conformal block function is associated either with an exchange matrix $A_{i i+1}$ or with its inverse depending on the sign of $u_{i}-u_{i+1}$. Every such matrix should satisfy Eq. (4). The fact that general permutations of the lightcone coordinates of a conformal block can be effected by inequivalent products of transpositions, but must induce a unique analytic continuation, imposes strong consistency conditions on the numerical exchange algebra coefficients.

Consider a set of $n$-point conformal blocks $F_{\alpha}\left(\ldots, u_{i}, u_{i+1}, u_{i+2}, \ldots\right)$ at a configuration $u_{i}>u_{i+1}>u_{i+2}$. The transposition of a pair of neighbouring coordinates is described by two exchange matrices $A_{i} \equiv A_{i i+1}$ and $A_{i+1} \equiv A_{i+1 i+2}$ with entries determined by the bilinear exchange algebra relations for the intertwining operators involved:

$$
\begin{aligned}
& F_{\alpha}\left(\ldots u_{i+1}, u_{i}, u_{i+2} \ldots\right)=\eta \sum_{\beta}\left(A_{i}\right)_{\alpha \beta} F_{\beta}\left(\ldots u_{i}, u_{i+1}, u_{i+2} \ldots\right), \\
& F_{\alpha}\left(\ldots u_{i}, u_{i+2}, u_{i+1} \ldots\right)=\eta \sum_{\beta}\left(A_{i+1}\right)_{\alpha \beta} F_{\beta}\left(\ldots u_{i}, u_{i+1}, u_{i+2} \ldots\right) .
\end{aligned}
$$

The ordering of the three coordinates can be reversed by applying either $A_{i}$ first, then $A_{i+1}$, then $A_{i}$, or $A_{i+1}$ first, then $A_{i}$, then $A_{i+1}$. For the result to be unique it is thus necessary and sufficient that

$$
A_{i} A_{i+1} A_{i}=A_{i+1} A_{i} A_{i+1} .
$$

We shall abbreviate this type of matrix equation by the notation $A_{i} * A_{i+1}$. Exchange matrices describing the transpositions of disjoint pairs of neighbouring coordinates commute obviously. This algebra of $n-1$ matrices $A_{i}$ is known as the braid algebra $3 n[9]$. 
The first issue describing an exchange algebra (e.g. the size of matrices Eq. (12)) is a "scheme" that tells among which pairs of $T$-modules the field under consideration interpolates. This scheme determines the general structure of the consistency conditions to solve. To be specific, we shall in a moment discuss a typical scheme which occurs in every one of the minimal theories of [1]. The result will be that there is only a finite number of consistent algebras for this scheme. If this holds true (we don't doubt this) for every (finite) scheme, the problem to find a local field with different schemes of interpolation on the two light-cones is highly nontrivial in view of Eq. (3). On the other hand, nonscalar primary fields should be of this type. We believe that this problem is related to the question of nonscalar primary fields contributing to "nondiagonal" modular invariant partition functions [4].

The scheme we want to discuss explicitly is the following. We assume a finite number $q-1$ of Hilbert space sectors $\mathscr{H}_{1}=$ vacuum sector, $\mathscr{H}_{2}, \ldots, \mathscr{H}_{q-1}$ and a primary field $\Phi$ interpolating only between neighbouring sectors: $\Phi \mathscr{H}_{i} \subset \mathscr{H}_{i-1} \oplus \mathscr{H}_{i+1}$. In a minimal theory [1] with central charge $c=c(p, q)=1$ $-6(q-p)^{2} / q p$ this is the field $\Phi_{1,2}$ of dimensions $h=\bar{h}=h_{1,2}(c)$, and $\mathscr{H}_{i}$ are the sectors with $L_{0} \bmod \mathbb{Z}=\bar{L}_{0} \bmod \mathbb{Z}=h_{1, i}(c), h_{n, m}(c)$ being given by the Kac formula. We shall, however, not refer to this particular realization of our assumptions.

According to the spirit of the preceding section, we consider $\mathscr{H}_{i}=V_{i} \otimes V_{i}$ as the direct product of two light-cone $T, \bar{T}$-modules and introduce intertwining lightcone operators $a_{i}: V_{i} \rightarrow V_{i+1}$ and $a_{i}^{+}, i=1, \ldots, q-2$. The complete exchange algebra is then parametrized by the following relations:

$$
\begin{aligned}
& a_{1}^{+}\left(u^{\prime}\right) a_{1}(u) \quad=\eta a_{1}^{+}(u) a_{1}\left(u^{\prime}\right) \\
& a_{i+1}\left(u^{\prime}\right) a_{i}(u) \quad=\eta \omega_{i} a_{i+1}(u) a_{i}\left(u^{\prime}\right) \\
& \left.\left(\begin{array}{c}
a_{i}\left(u^{\prime}\right) a_{i}^{+}(u) \\
a_{i+1}^{+}\left(u^{\prime}\right) a_{i+1}(u)
\end{array}\right)=\eta\left(\begin{array}{cc}
\alpha_{i} & \beta_{i} \\
\gamma_{i} & \delta_{i}
\end{array}\right)\left(\begin{array}{c}
a_{i}(u) a_{i}^{+}\left(u^{\prime}\right) \\
a_{i+1}^{+}(u) a_{i+1}\left(u^{\prime}\right)
\end{array}\right)\right\} i=1, \ldots, q-3 \\
& a_{q-2}\left(u^{\prime}\right) a_{q-2}^{+}(u)=\eta \tilde{\omega} a_{q-2}(u) a_{q-2}^{+}\left(u^{\prime}\right) \text { for } u>u^{\prime} \text {. }
\end{aligned}
$$

We shall assume all exchange matrices to be symmetric, i. e. $\gamma_{i}=\beta_{i}$. Since, as it turns out, the consistency conditions (14) impose restrictions only on the products $\beta_{i} \gamma_{i}$, the full generality can be recovered in the end by similarity transformations with diagonal matrices. These must (and can) be chosen in agreement with Eq. (4).

The complex phases $\eta$ and $\omega_{i}$ are related with the dimensions of the fields involved (see Sect. 5). $\eta$ is not relevant for the consistency. We shall consider $\omega=\omega_{1}$ as a so far free phase parameter, and determine all remaining coefficients as functions of $\omega$. The requirement of a finite number of sectors will in the end quantize the parameter $\omega$, and thus the dimensions.

Due to the selection rule of matching initial and final $T$-modules for the exchange algebra, all consistency conditions Eq. (14) can be discussed within the sets of trilinear products of intertwining operators with common initial and final $T$-modules. Select a set of nonvanishing products of three light-cone operators $a, b, c$ out of $\left\{a_{i}, a_{i}^{+} \mid i=1, \ldots, q-1\right\}$ with common initial and final $T$-modules, residing at points $u>u^{\prime}>u^{\prime \prime}: a^{(\alpha)}(u) b^{(\alpha)}\left(u^{\prime}\right) c^{(\alpha)}\left(u^{\prime \prime}\right), \alpha=1, \ldots, r$. The transposition of neighbouring coordinates is described via the exchange algebra in terms of two $r \times r$ 
exchange matrices which must be read off Eq. (15):

$$
\begin{aligned}
& a^{(\alpha)}\left(u^{\prime}\right) b^{(\alpha)}(u)=\eta \sum_{\beta}\left(A_{12}\right)_{\alpha \beta} a^{(\beta)}(u) b^{(\beta)}\left(u^{\prime}\right), \\
& b^{(\alpha)}\left(u^{\prime \prime}\right) c^{(\alpha)}\left(u^{\prime}\right)=\eta \sum_{\beta}\left(A_{23}\right)_{\alpha \beta} b^{(\beta)}\left(u^{\prime}\right) c^{(\beta)}\left(u^{\prime \prime}\right) .
\end{aligned}
$$

The inversion of the ordering of the three coordinates gives a unique result, as has been argued above, provided

$$
A_{12} A_{23} A_{12}=A_{23} A_{12} A_{23} \text {, i.e. } A_{12} * A_{23} \text {. }
$$

This set of consistency conditions (one for each pair of initial and final $T$-modules) can be solved explicitly. The independent conditions arise from the following cases:

a) $a b c: \quad V_{i} \rightarrow V_{i+3} ; i=1, \ldots, q-4$. There is only one operator product $a_{i+2} a_{i+1} a_{i}$, and the exchange "matrices" are $A_{12}=\omega_{i+1}, A_{23}=\omega_{i}$.

B) $a b c: V_{1} \rightarrow V_{2}$. There are two products $a_{1} a_{1}^{+} a_{1}, a_{2}^{+} a_{2} a_{1}$ and the exchange matrices are

$$
A_{12}=\left(\begin{array}{ll}
\alpha_{1} & \beta_{1} \\
\beta_{1} & \delta_{1}
\end{array}\right), \quad A_{23}=\left(\begin{array}{ll}
1 & \\
& \omega_{1}
\end{array}\right) \text {. }
$$

$\gamma) a b c: V_{i} \rightarrow V_{i+1} ; i=2, \ldots, q-3$. There are three products $a_{i} a_{i-1} a_{i-1}^{+}, a_{i} a_{i}^{+} a_{i}$,

$$
a_{i+1}^{+} a_{i+1} a_{i}, \quad \text { and } A_{12}=\left(\begin{array}{lll}
\omega_{i-1} & & \\
& \alpha_{i} & \beta_{i} \\
& \beta_{i} & \delta_{i}
\end{array}\right), \quad A_{23}=\left(\begin{array}{lll}
\alpha_{i-1} & \beta_{i-1} & \\
\beta_{i-1} & \delta_{i-1} & \\
& & \omega_{i}
\end{array}\right) \text {. }
$$

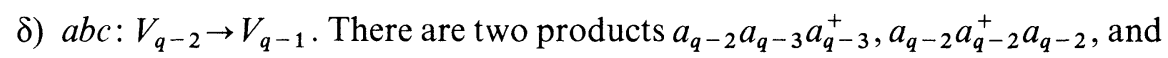

$$
A_{12}=\left(\begin{array}{cc}
\omega_{q-3} & \\
& \tilde{\omega}
\end{array}\right), \quad A_{23}=\left(\begin{array}{cc}
\alpha_{q-3} & \beta_{q-3} \\
\beta_{q-3} & \delta_{q-3}
\end{array}\right) \text {. }
$$

The conditions are solved as follows.

a) $\omega_{i+1} * \omega_{i}$ is equivalent to $\omega_{i+1}=\omega_{i}$, hence $\omega_{1}=\omega_{2}=\ldots=\omega_{q-3}=\omega$.

$\beta)\left(\begin{array}{cc}\alpha_{1} & \beta_{1} \\ \beta_{1} & \delta_{1}\end{array}\right) *\left(\begin{array}{cc}1 & \\ & \omega\end{array}\right)$ is solved by

$$
\left(\begin{array}{ll}
\alpha_{1} & \beta_{1} \\
\beta_{1} & \delta_{1}
\end{array}\right)=\frac{1}{1-\omega}\left(\begin{array}{cc}
-\omega^{2} & \sqrt{-\omega+\omega^{2}-\omega^{3}} \\
\sqrt{-\omega+\omega^{2}-\omega^{3}} & 1
\end{array}\right)
$$

(The second solution $A_{12}=A_{23}$ is in conflict with $\gamma$ ) or $\delta$ ) unless $\omega=1$. Then, however, all exchange matrices become trivial.)

$\gamma)\left(\begin{array}{lll}\omega & & \\ & \alpha_{i} & \beta_{i} \\ & \beta_{i} & \delta_{i}\end{array}\right) *\left(\begin{array}{ccc}\alpha_{i-1} & \beta_{i-1} & \\ \beta_{i-1} & \delta_{i-1} & \\ & & \omega\end{array}\right)$ is an overspecified nonlinear system for $\alpha_{i}, \beta_{i}, \delta_{i}$. It is self-consistent if and only if $\left(\begin{array}{cc}\alpha_{i-1} & \beta_{i-1} \\ \beta_{i-1} & \delta_{i-1}\end{array}\right)$ has an eigenvalue $\lambda=\omega$. Then it implies that $\left(\begin{array}{cc}\alpha_{i} & \beta_{i} \\ \beta_{i} & \delta_{i}\end{array}\right)$ has an eigenvalue $\lambda=\omega$, too. Moreover, $\alpha_{i}+\delta_{i}$ $=\alpha_{i-1}+\delta_{i-1}$. Since spectrum $\left(\begin{array}{cc}\alpha_{1} & \beta_{i} \\ \beta_{1} & \delta_{1}\end{array}\right)=\{1, \omega\}$, the system is solvable recursively, 
and spectrum $\left(\begin{array}{cc}\alpha_{i} & \beta_{i} \\ \beta_{i} & \delta_{i}\end{array}\right)=\{1, \omega\}$. Explicitly we find

$\left(\begin{array}{cc}\alpha_{i} & \beta_{i} \\ \beta_{i} & \delta_{i}\end{array}\right)=\frac{1}{s_{i}}\left(\begin{array}{cc}-(-\omega)^{i+1} & \sqrt{-\omega s_{i-1} s_{i+1}} \\ \sqrt{-\omega s_{i-1} s_{i+1}} & 1\end{array}\right), \quad$ where $s_{i}=\sum_{p=0}^{i}(-\omega)^{p}$.

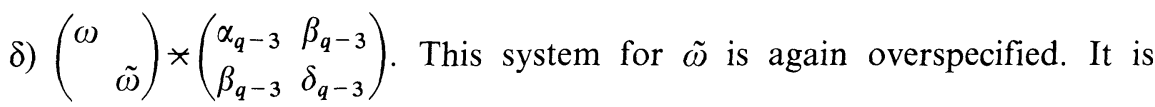
solvable if i) $\left(\begin{array}{ll}\alpha_{q-3} & \beta_{q-3} \\ \beta_{q-3} & \delta_{q-3}\end{array}\right)$ has an eigenvalue $\lambda=\omega$, and if ii) $\left(\alpha_{q-3}+\delta_{q-3}\right.$ $-\omega)\left(\alpha_{q-3} \delta_{q-3}+\left(\delta_{q-3}-\omega\right)^{2}\right)=0$. The condition i) is satisfied (see above), while ii) is equivalent to the break-off condition

$$
s_{q-1} \equiv 1-\omega+\omega^{2} \ldots+(-\omega)^{q-1}=0
$$

Then $\tilde{\omega}=1$.

Equation (19) is solved by

$$
\omega=-e^{-2 \pi i p / q}=e^{-i \pi \frac{2 p-q}{q}},
$$

where $p$ and $q$ have no common divisors. Otherwise, some $s_{i}$ occurring in Eq. (18) in the denominator would vanish.

The conditions (4) have not yet been imposed. Indeed, writing $s_{i}=(-\omega)^{i / 2} \sin (\pi(i+1) p / q) / \sin (\pi p / q)$, it is easily seen, that the symmetric solutions (18) satisfy Eq. (4) if and only if $\sin \pi i p / q \cdot \sin \pi(i+2) p / q>0$. The general, nonsymmetric solution

$$
\left(\begin{array}{ll}
\alpha_{i} & \beta_{i} \\
\gamma_{i} & \delta_{i}
\end{array}\right)=\frac{1}{s_{i}}\left(\begin{array}{cc}
-(-\omega)^{i+1} & t_{i}^{-1} \sqrt{-\omega s_{i-1} s_{i+1}} \\
t_{i} \sqrt{-\omega s_{i-1} s_{i+1}} & 1
\end{array}\right)
$$

satisfies Eq. (4) with real $t_{i}$ if $\sin \pi i p / q \cdot \sin \pi(i+2) p / q>0$, and with imaginary $t_{i}$ if $\sin \pi i p / q \cdot \sin \pi(i+2) p / q<0$. In the latter case it is impossible to satisfy Eqs. (3) and (5) at the same time. Hence, if we want the field $\Phi$ to be hermitian and scalar, $\sin \pi i p / q$ and $\sin \pi(i+2) p / q$ must always have the same sign, i.e.

$$
p= \pm 1 \bmod q \text {. }
$$

Since all exchange matrices have only eigenvalues $\lambda=1$ and $\lambda=\omega$, the $q^{\text {th }}$ power of all exchange matrices is the identity matrix. This fact is important for the monodromy properties of conformal block functions, since the analytic continuation of one coordinate once around another one is described by the square of the corresponding exchange matrix.

This completes the analysis of the exchange algebras consistent with the scheme of "nearest neighbour" interpolation among the sectors $\mathscr{H}_{1}, \ldots, \mathscr{H}_{q-1}$. In the next section we shall associate various models with these exchange algebras. The question, whether an arbitrary scheme possesses a consistent exchange algebra at all, should be discussed in the context of classification of braid algebras. 


\section{Relations to Known Models}

The minimal models of [1] with $c=c(p, q)=1-6(q-p)^{2} / q p$, where $p$ and $q$ have no common divisors, are known to contain a scalar field $\Phi=\Phi_{2}$, interpolating, according to the scheme assumed in the preceding section, among sectors $\mathscr{H}_{i}$ generated by fields $\Phi_{i}$ with dimensions $d_{i}=h_{1, i}(c)$. Hence, the exchange algebra of $\Phi_{2}$ must be contained in the solutions Eqs. (18), (20). Indeed, the 4-point conformal blocks of $\left\langle\Phi_{2} \Phi_{2} \Phi_{2} \Phi_{2}\right\rangle$ are hypergeometric functions, the analytic continuation behaviour of which is well known. It is exactly given by the coefficients $\alpha_{1}, \beta_{1}=\gamma_{1}, \delta_{1}$.

Obviously, another minimal model with $p^{\prime}=p \bmod q$ has the same exchange algebra, since the parameter $\omega$ is the same. We shall see that the family of exchange algebras (15), (18), (20) is even relevant for models with $c>1$. Let us therefore discuss how to extract more detailed information about a model out of its exchange algebra.

Assume first that the field $\Phi=\Phi_{2}$ has a nonvanishing 2-point function (i.e. it carries no internal symmetries). The $u$ conformal block $\left\langle a_{1}^{+}\left(u_{1}\right) a_{1}\left(u_{2}\right)\right\rangle$ of this 2 point function is $\left(u_{12}-i \varepsilon\right)^{-2 d_{2}}$ which changes by a phase factor $e^{i \pi 2 d_{2}}$ when $u_{12}>0$ is analytically continued to $-u_{12}$. Then Eq. (15) tells us that

$$
\eta=e^{i \pi 2 d_{2}} \text {. }
$$

Now consider the 3-point function $\left\langle\Phi_{3} \Phi_{2} \Phi_{2}\right\rangle$ where the field $\Phi_{3}$ generates $\mathscr{H}_{3}$. Its $u$ conformal block $\left\langle a_{0 h_{3}}^{\left(\Phi_{3}\right)}\left(u_{1}\right) a_{2}\left(u_{2}\right) a_{1}\left(u_{3}\right)\right\rangle$ is proportional to $\left(u_{23}-i \varepsilon\right)^{-2 d_{2}+d_{3}}$, which changes by a phase factor $e^{i \pi\left(2 d_{2}-d_{3}\right)}$ when $u_{23}>0$ is analytically continued to $-u_{23}$. Again Eq. (15) tells us that

$$
\eta \omega=e^{i \pi\left(2 d_{2}-d_{3}\right)}, \quad \text { i.e. } \omega=e^{-i \pi d_{3}} .
$$

We thus obtain from Eq. (20) the dimension $d_{3}=(2 p-q) / q \bmod 2$ of the field $\Phi_{3}$. Next, consider the 4-point function $\left\langle\Phi_{2} \Phi_{2} \Phi_{2} \Phi_{2}\right\rangle$. Both its conformal blocks $F_{1}=\left\langle a_{1}^{+} a_{1} a_{1}^{+} a_{1}\right\rangle$ and $F_{2}=\left\langle a_{1}^{+} a_{2}^{+} a_{2} a_{1}\right\rangle$ are, except for a common factor $\left(u_{12} u_{34}\right)^{-2 d_{2}}$, real functions of the variable $x=u_{12} u_{34} / u_{13} u_{24}$ in the interval $0<x<1$ [8]. Exchanging $u_{2} \leftrightarrow u_{4}$ takes $x$ into $1-x$, where $F_{1}, F_{2}$ are again real, except for a phase $\eta$ acquired by the inhomogeneous factor. This exchange is described by the matrix product $\eta^{3} A_{23} A_{12} A_{23}$ (with $A_{12}, A_{23}$ from $\beta$ ) in the preceding section). Then $\eta^{2} A_{23} A_{12} A_{23}$ must be real, i. e.

$$
\eta^{2} \omega^{2} /(1-\omega) \in \mathbb{R} \text { and } \sqrt{1+\omega-\omega^{-1}}=\sqrt{1+2 \cos 2 \pi p / q} \in \mathbb{R}
$$

We thus obtain the dimension $d_{2}=(3 p-2 q) / 4 q \bmod 1 / 4$ of the field $\Phi_{2}$, as well as some restriction on $p$, which is fulfilled if Eq. (21) is satisfied. Finally, consider the 3-point function $\left\langle\Phi_{i+1} \Phi_{i} \Phi_{2}\right\rangle$, where $\Phi_{i}$ are the fields generating $\mathscr{H}_{i}$. It is proportional to $\left(u_{23}-i \varepsilon\right)^{d_{2}+1-d_{l}-d_{2}}$ which acquires a phase $e^{-i \pi\left(d_{i+1}-d_{i}-d_{2}\right)}$ if $u_{23}>0$ is continued to $-u_{23}$. By a naive cluster idea we represent the intertwining light-cone operators $a_{j}^{(i)}: V_{j} \rightarrow V_{j+i-1}$ belonging to $\Phi_{i}$ as some local limit of products $a_{j+i-2} \ldots a_{j+1} a_{j}$ of intertwining operators belonging to $\Phi_{2}$. Then we can compute by repeated use of Eq. (15) the exchange relation for $u>u^{\prime}$,

$$
a_{j+i-1}\left(u^{\prime}\right) a_{1}^{(i)}(u)=(\eta \omega)^{i-1} a_{2}^{(i)}(u) a_{1}\left(u^{\prime}\right) \text {. }
$$


The exchange operation changes the function $\left\langle\Phi_{i+1} \Phi_{i} \Phi_{2}\right\rangle$ into the different function $\left\langle\Phi_{i+1} \Phi_{2} \Phi_{i}\right\rangle$. In order to relate the analytic exchange phase with the known phase of either of these functions, we must study the monodromy operation, which is the square of the exchange operation and takes either function into itself. Comparing the monodromy phases

$$
(\eta \omega)^{2(i-1)}=e^{-2 \pi i\left(d_{i+1}-d_{i}-d_{2}\right)}
$$

yields $d_{i+1}-d_{i}=(i-1)\left(d_{3}-2 d_{2}\right)+d_{2}$ mod1, which leads recursively to the dimensions $d_{i}=(i-1)\left((i-2) d_{3} / 2-(i-3) d_{2}\right) \bmod 1$ of the fields $\Phi_{i}$. If we ignore for the moment all " $\bmod y$ " reservations, this is

$$
d_{i}=(i-1)((i+1) p-2 q) / 4 q,
$$

which coincides exactly with the Kac formula values $d_{i}=h_{1, i}(c)$ for a minimal model with $c=c(p, q)$. The dimensions shifted by integers presumably just belong to secondary fields (which have the same exchange algebras as the primary fields). We do not know, whether values of $d_{2}$, and thus of $d_{i}$, shifted by non-integer multiples of $1 / 4$, belong to any meaningful models.

Let us now discuss the case that $\Phi_{2}$ has an internal symmetry. To be specific, assume that $\Phi_{2}$ is an $S U(2)$ doublet. Clearly, the exchange algebra must be completed by $S U(2)$ selection rules, but a solution to the consistency conditions which is a Clebsch-Gordan extension of Eqs. (15), (18), (20) can be found [10], if $\Phi_{i}$ is assumed to carry isospin $j=(i-1) / 2$. However, in this case the relation of the parameters to the dimensions is a different one. Since only the antisymmetric product of the $S U(2)$ doublet $\Phi_{2}$ can have a nonvanishing 2-point function, we have this time

$$
\eta=-e^{i \pi 2 d_{2}} .
$$

The symmetric product enters the 3-point function $\left\langle\Phi_{3} \Phi_{2} \Phi_{2}\right\rangle$, hence

$$
\eta \omega=e^{i \pi\left(2 d_{2}-d_{3}\right)}, \quad \text { i.e. } \omega=-e^{-i \pi d_{3}} .
$$

The reality requirement reads again

$$
\eta^{2} \omega^{2} /(1-\omega) \in \mathbb{R}, \quad \text { and } \quad \cos 2 \pi p / q>-\frac{1}{2},
$$

and a similar cluster idea as above gives again

$$
(\eta \omega)^{2(i-1)}=e^{-i \pi 2\left(d_{i+1}-d_{i}-d_{2}\right)} .
$$

From these relations we compute, similarly as before, $d_{3}=2 p / q \bmod 2$, $d_{2}=3 p / 4 q \bmod 1 / 4$, and recursively $d_{i}=(i-1)\left((i-2) d_{3} / 2-(i-3) d_{2}\right) \bmod 1$. If we ignore again the "mod $y$ " reservations, we obtain

$$
d_{i}=j(j+1) \cdot p / q\left(j=\frac{i-1}{2}\right),
$$

which are the well known values for the $S U(2)$ symmetric models with current algebra of level $k=q-2$, if $p=1$. Such models have $c>1$.

Finally we want to mention a relation of the braid algebra (17) with the YangBaxter algebra of a "solid-on-solid" (SOS) model of statistical mechanics [11]. The general existence of such relations was suggested, too, by Fröhlich [6]. The 
dependence of the Yang-Baxter matrices on an additive variable $u$ can be eliminated, in order to establish an identification with the constant braid algebra matrices, in the limit $u \rightarrow i \infty$, provided the elliptic module $K=\pi / 2$. Our solutions (18) can be identified (associating an intertwining operator $a_{i}$ with a link between two plaquettes of "heights" $i, i+1)$ with the symmetrized Boltzmann weights of [11], if the parameters of the SOS model are set $w_{0}=0, e^{4 i n}=-\omega$. The break-off condition (19) corresponds to the "restricted SOS" (RSOS) model with $w_{q}=0$, and its solution (20) means $v \equiv K / \eta=q /(q-p)$. However, only $p= \pm 1 \bmod q$ avoids the appearance of negative or imaginary Boltzmann weights.

\section{Discussion}

The exchange algebra described in this paper must be completed in two points. The first point is the introduction of further relations describing the commutation of different local fields among each other. This completion is straightforward in its general structure, dictated by the $Z$ selection rules. There are again consistency conditions in the form of a braid algebra. For the Ising model we have checked that these fix the additional structure constants uniquely. We conjecture that it sufficient in every model to establish the consistency of the exchange algebra among intertwining operators belonging to some "fundamental" field only, considering the remaining fields as "composite" fields. The consistency of the exchange algebra relevant for these composite fields would then be a consequence of the consistency for the fundamental field. Our conjecture is supported by the existence of a field in every minimal model (e.g. the field with dimension $h_{2,2}(c)$ if $p q$ is even), which under repeated application of the operator product expansion generates fields with all other light-cone dimensions of the model.

The second point is that the exchange algebra in its present form does not specify the singularity at coinciding light-cone points $u^{\prime}=u$. In particular, at $u^{\prime} \neq u$ the algebra of secondary fields is indistinguishable from that of the corresponding primary fields; e.g. the light-cone component $T(u)$ of the energy-momentum tensor field simply commutes with every intertwining operator at $u^{\prime} \neq u$.

Leaving aside these deficiencies, we believe that the exchange algebra is the most fundamental structure inherent in two-dimensional conformal quantum field theory. It is even stronger than the Virasoro algebra, since it provides the algebraic "missing link" among the different Virasoro representation sectors of Hilbert space. The consistency conditions of the exchange algebra are matrix representations of a braid algebra. A classification of conformal theories with a finite number of primary fields along the lines of classification of braid algebra representations promises an interesting new glimpse into the beauty of two dimensional conformal quantum field theory.

Acknowledgements. I am very much indebted to B. Schroer and M. Karowski for many discussions and suggestions. 


\section{References}

1. Belavin, A.A., Polyakov, A.M., Zamolodchikov, A.B.: Infinite conformal symmetry in twodimensional quantum field theory. Nucl. Phys. B241, 333 (1984)

2. Rehren, K.-H., Schroer, B. : Exchange algebra on the light-cone and order/disorder $2 n$-point functions in the Ising field theory. Phys. Lett. B 198, 84 (1987)

3. Rocha-Caridi, A.: Vacuum vector representations of the Virasoro algebra. In: Vertex operators in mathematics and physics. Lepowsky, J., Mandelstam, S., Singer, I.M. (eds.). p. 451. Berlin, Heidelberg, New York: Springer 1984

4. Cardy, J.: Operator content of two-dimensional conformally invariant theories. Nucl. Phys. B270, 186 (1986)

Capelli, A., Itzykson, C., Zuber, J.B.: Modular invariant partition functions in two dimensions. Nucl. Phys. B280, 445 (1987)

5. Schroer, B., Swieca, J.A.: Conformal transformations for quantized fields, Phys. Rev. D10, 480 (1974)

Schroer, B., Swieca, J.A., Völkel, A.H.: Global operator expansions in conformally invariant relativistic quantum field theory. Phys. Rev. D11, 1509 (1975)

6. Fröhlich, J. : Statistics of fields, the Yang-Baxter equation, and the theory of knots and links. To appear in Cargèse 1987 proceedings

7. Lüscher, M., Mack, G. : Global conformal invariance in quantum field theory. Commun. Math. Phys. 41, 203 (1975)

8. Rehren, K.-H., Schroer, B.: Quasiprimary fields: An approach to positivity of $2 D$ conformal quantum field theory. To appear in Nucl. Phys. B

9. Artin, E.: Collected papers, pp. 416-498. Lang, S., Tate, J.T. (eds.). Reading, MA 1965

10. Schroer, B.: New methods and results in conformal $Q F T_{2}$ and the 'string idea'. CERN preprint TH 4850/87; to appear in Cargèse 1987 proceedings

11. Andrews, G.E., Baxter, R.J., Forrester, P.J. : Eight vertex SOS model and generalized RogersRamanujan-type identities. J. Stat. Phys. 35, 193 (1984)

Communicated by R. Haag

Received October 10, 1987 